\title{
VALIDADE PREDITIVA DO MOVEMENT ASSESSMENT OF INFANTS PARA CRIANÇAS PRÉ-TERMO BRASILEIRAS
}

\author{
Ana Amélia Cardoso ${ }^{\mathrm{E}}$; Lívia C. Magalhães ${ }^{T}$, Regina Helena C. Amorim $^{\mathrm{P}}$, \\ Maria Lúcia PaixãoF*, Marisa C. Mancini' ${ }^{\top}$, Luciana D.F. Rossi ${ }^{H}$
}

\begin{abstract}
RESUMO - A validade preditiva da Avaliação do Movimento do Bebê(M ovement Assessment of Infants- MAI), para detecção precoce de paralisia cerebral, foi analisada em 89 crianças brasileiras nascidas com idade gestacional $\leq 32$ semanase peso $\leq 1500 \mathrm{~g}$. O MAI foi aplicado aos 4 e 8 meses de idade corrigida e as crianças foram submetidas a avaliação neurológica entre 2 e 7 anos de idade. Foram calculadas estimativas de sensibilidade $(0,61$ a 1,0$)$, especificidade $(0,79$ a 0,95$)$, valor de predição positiva $(0,48$ a 0,73$)$ e valor de predição negativa $(0,79$ a 1,0$)$, aos 4 e 8 meses, para escores $\geq 13$ e $\geq 10$ pontos de risco. Os melhores índices preditivos foram obtidos aos 8 meses e para o ponto de corte $\geq 13$ pontos de risco. Um critério menos restritivo ( $\geq 10$ pontos) pode ser útil para predição de transtornos da coordenação motora, na idade escolar.
\end{abstract}

PALAVRAS-CHAVE: prematuridade, detecção precoce, desenvolvimento infantil, paralisia cerebral, validade preditiva, MAI.

\begin{abstract}
Predictive validity of the Movement Assessment of Infants (MAI) for Brazilian preterm children
ABSTRACT - The predictive validity of the Movement Assessment of Infants (MAI) for the detection of cerebral palsy was analyzed in 89 Brazilian infants, born with gestational age $\leq 32$ weeks and weight $\leq 1500 \mathrm{~g}$. The infantswere assessed with the MAI at 4 and 8 months, corrected ages, and were submitted to a neurological evaluation between the ages 2 and 7 years old. Estimates of sensibility $(0.61-1.0)$, specificity $(0.79-0.95)$, positive predictive value $(0.48-0.73)$ and negative predictive value $(0.79-1.0)$ were calculated at 4 and 8 months, for risk points $\geq 13$ and $\geq 10$. The best predictive values were obtained at 8 months, with a cut off $\geq 13$ risk points. A less restrictive criteria ( $\geq 10$ points) might be useful for the prediction of motor coordination problems at school age.
\end{abstract}

KEY WORDS: prematurity, early detection, child development, cerebral palsy, predictive validity, MAI.

O aumento nastaxas de sobrevivência de recémnascidos de risco estimula o interesse pelo impacto de fatores, como a prematuridade, no desenvolvimento cognitivo, neuromotor e socioemocional da criança ${ }^{1}$. Dentre as possíveis conseqüências da prematuridade está a paralisia cerebral (PC), que provoca grandes demandas, não só em termos de serviços de saúde, educação e assistência social, mas também para as famílias e as próprias crianças. Estudos preditivos do diagnóstico da $\mathrm{PC}^{2-11}$ enfatizam a relação entre prematuridade e baixo peso ao nascimento com o diagnóstico de PC e recomendam que recém-nascidos pré-termo, com muito baixo peso ou que sofreram intercorrênciasneurológicas neonatais, sejam acompanhados desde o nascimen- to ${ }^{12}$, para prevenir possíveis atrasos ou tratar, precocemente, as seqüelas mais severas.

Dada a importância da detecção da PC, além do exame neurológico, foram criados vários testes para diagnóstico precoce ${ }^{13-17}$. Na área de Fisioterapia e Terapia Ocupacional, um dos instrumentos mais conhecidos é a Avaliação do Movimento do Bebê (Movement Assessment of Infants-MAI), de Chandler, Andrews e Swanson ${ }^{18}$, teste usado em programas de acompanhamento nos EstadosUnidos, e submetido a vários estudos de validade e confiabilidade. No primeiro estudo, Chandler e colaboradores ${ }^{18}$ conduíram que bebêscom total de pontos de risco de 0 a 7 apresentavam baixo risco para PC. A pontuação de 8 a 13 seria indicativa de risco mé-

Departamentos de Terapia Ocupacional ${ }^{\top}$ (DTO), Fisioterapia (DFIT) e Pediatria ${ }^{\mathrm{P}}$ (PED) da Universidade Federal de Minas Gerais, Belo Horizonte MG, Brasil (UFMG); ' Departamento de Fisioterapia do Hospital das Clínicas/UFM G; ETerapeuta Ocupacional, Bolsista de

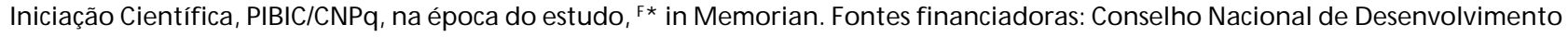
Científico e Tecnológico - CNPq e Fundação de Apoio à Pesquisa de Minas Gerais - FAPEMIG.

Recebido 11 Dezembro 2003, recebido na forma final 10 Maio 2004. Aceito 9 Julho 2004.

Dra. Lívia C. Magalhães - Rua Chicago 337/41 - 30315-520 Belo Horizonte MG - Brasil. E-mail: liviam@gcsnet.com.br. 
dio e acima de 13 pontos, de alto risco. Em estudo subseqüente, Schneider, Lee e Chasnoff ${ }^{19}$ observaram que 0 a 7 pontos de risco são insuficientes para identificar desenvolvimento neuromotor normal, por isso sugerem como critério de risco resultados "acima de 10 pontos". Osíndices de confiabilidade reportados para o MAI, variam de 0,72 a 0,91 , entre examinadores, e de 0,76 a 0,79 para teste-reteste $^{18,20}$. Haley e colaboradores ${ }^{21}$ questionam a confiabilidade de algunsitensindividuais do teste, especialmente na área de avaliação de reflexos primitivos, a qual requer maior manuseio físico da criança.

Como indicado por Campbell ${ }^{22}$, a sensibilidade do MAl aos 4 meses, na previsão do diagnóstico de PC, foi $73,5 \%$ e a especificidade $62,7 \%$. Swanson e colaboradores ${ }^{5}$ observaram que a sensibilidade do MAl é alta ( $83 \%$ e $96 \%$ aos 4 e aos 8 meses), mas a especificidade é relativamente baixa ( $72 \%$ e $59 \%$, para as respectivas idades). Washington e Deitz $^{23}$ apontam sensibilidade de $81 \%$, especificidade de $56 \%$, com número elevado $(44 \%)$ de falsos positivos, aos 6 meses de idade. Visando melhorar os valores de validade preditiva do teste, alguns autores exploraram diferentes pontos de corte, ou número de pontos de risco no MAI, para detecção de PC. Swanson e colaboradores ${ }^{5}$ tomaram como critério número de pontos de risco $\geq 10$ para detecção de PC, reportando, para o MAI de 4 meses, sensibilidade de $88 \%$ e especificidade de $78 \%$. Darrah e colaboradores ${ }^{24}$ adotaram pontuação $\geq$ 9 e encontraram sensibilidade de 72,7; especificidade de 93,0; valor de predição positiva de 58,3 e valor de predição negativa de 95,7. Segundo esses autores ${ }^{24}$, o MAI seria o melhor teste para detecção de PC aos 4 meses.

Fetterse Tronick ${ }^{25}$ compararam o poder preditivo do AIMS - Alberta Infant Motor Scale ${ }^{26}$ com o do MAl e observaram que, para ambos os testes, a melhor combinação de sensibilidade e especificidade ocorreu aos 7 meses. Além disso, um ponto de corte maior que 9 pontos de risco, no MAI, ou o percentil 2, no AIMS, ofereceram a melhor combinação de valoresna predição de atraso motor aos 15 meses. O MAI aplicado aos 7 meses apresentou sensibilidade razoável $(0,75)$, porém baixa especificidade, quando se utilizou 9 pontos de risco como ponto de corte.

Segundo Campbell22, as limitações do MAI incluem muitositens com baixa confiabilidade entre examinadores e a falta de uma base psicométrica segura, pois não foram desenvolvidas normas de desempenho por idade, apenas perfis de risco para o $4^{\circ}, 6^{\circ}$ e $8^{\circ}$ mês. Não foi feita, também, demonstração da validade de construto, da sensibilidade e especificidade em amostras aleatórias. A maioria dos estudos sobre o MAI foi realizada com amostras altamente selecionadas, o que dificulta a generalização dos resultados para outras populações e outros países. Um dado importante é que o MAI foi criado há mais de 20 anos, com base na abordagem neuromaturacional, com pouca ênfase na observação da movimentação espontânea, a qual parece ter maior valor preditivo para desfechosfuturos do desenvolvimento motor. Embora existam controvérsias e críticas a respeito da utilidade do MAI, a maioria dosestudoso aponta como um teste confiável e que, quando se considera valoresacima de 9 ou 10 pontos, tem boa validade preditiva, sendo útil no diagnóstico precoce de PC, em crianças norte-americanas. Não existem estudos sobre a validade preditiva do MAI para crianças brasileiras, porém um estudo preliminar sobre a relação entre fatores de risco para PC e escores do $\mathrm{MAl}^{12}$ indica seu potencial no diagnóstico precoce.

O objetivo do presente estudo foi examinar a validade preditiva do MAI no diagnóstico precoce de PC, em crianças nascidas prematuras e que fazemacompanhamento no Ambulatório da Criança de Risco - ACRIAR - serviço interdisciplinar de acompanhamento de recém-nascidos pré-termo do Hospital das Clínicas da Universidade Federal de Minas Gerais (UFMG).

\section{MÉTODO}

Participantes - Participaram do estudo 89 crianças, nascidas na maternidade do Hospital das Clínicas da UFMG, com idade gestacional $\leq 32$ semanas e peso ao nascimento $\leq 1500 \mathrm{~g}$ e que foram acompanhadas no ACRIAR, no período de janeiro de 1991 a maio de 2002. Foram incluídasno estudo todasas criançasavaliadaspelo MAl aos 4 e 8 meses de idade corrigida e que passaram por exame neurológico entre as idades de 2 e 7 anos.

Instrumentação - O MAl é um teste para avaliação do desenvolvimento neuromotor com 65 itens divididos em 4 seções: tônusmuscular, reflexos primitivos, reações automáticas e movimentos voluntários. Para cada item foi desenvolvida uma escala numérica que representa a evolução das respostas da criança, que podem ser normais ou questionáveis, de acordo com o esperado para a idade da criança. Nas quatro seções do teste, o examinador deve estar atento para a possibilidade de encontrar respostas assimétricase deve registrá-las em uma coluna especial, na folha de pontuação.

O teste não apresenta escores normativos, masforam criados perfis de risco para PC aos 4, 6 e 8 meses de ida$\mathrm{de}^{18,23}$. Nessas idades, para os escores questionáveis ou 
anormais são dados pontos de risco, que somados nas quatro categorias, resultam em pontos totais de risco. Quanto maior o número de pontos de risco, especialmente se acima de 10 pontos, pior é o prognóstico da criança.

Procedimentos - O MAl é aplicado como rotina no ACRIAR, aos 4 e 8 meses de idade corrigida, por fisioterapeutase terapeutas ocupacionaistreinadas de acordo com as instruções do manual de teste. A confiabilidade entre examinadores é estabelecida mediante avaliação conjunta como instrutor, de pelo menos 10 crianças, até que se atinja o mínimo de $80 \%$ de concordância entre os escores. Periodicamente, são feitas avaliações conjuntas para re-checagem da confiabilidade. Ascrianças são avaliadas na presença dos pais ou responsáveis, que participam ativamente da consulta, criando um ambiente adequado para a avaliação. Imediatamente após a testagem, osdadoscoletadossão organizadosem planilha Excel, no banco de dados do ACRIAR.

Cabe ressaltar que, na primeira consulta, os pais são esclarecidos sobre os objetivos do programa de acompanhamento e sobre as atividades de pesquisa do ACRIAR. Os pais de todas as crianças que participaram deste estudo assinaram um termo de consentimento para a utilização dos dados de seus filhos para fins de pesquisa. O projeto foi aprovado pelo Comitê de Ética em Pesquisa (COEP) da UFMG.

Das 558 crianças registradas no banco de dados do ACRIAR, 105 crianças atenderam aos quatro critérios para inclusão no estudo. Foram excluídas 15 criançasque abandonaram o programa ainda sem diagnóstico, e 1 criança que faleceu antes de completar 24 meses. A amostra final foi de 89 crianças, das quais 46 do sexo feminino e 43 do sexo masculino.

A validade preditiva do MAI foi estimada utilizando 4 índices ${ }^{17}$ : a) sensibilidade (S); b) especificidade (E); c) Valor de Predição Positiva (VPP), que é a probabilidade de a criança apresentar $\mathrm{PC}$, quando os escores de risco no MAI são maiores que o ponto de corte e d) Valor de Predição Negativa (VPN), que é a probabilidade de a criança não apresentar PC, quando os escores de risco no MAI são menoresque o ponto de corte. Geralmente, os índices não são analisados individualmente, mas observa-se qual a melhor combinação de sensibilidade / especificidade e valores de predição positiva e negativa. Neste estudo foram adotados os valores sugeridos por Glascoe e colaboradores ${ }^{27}$ para avaliar a validade preditiva de testes: 0,80 para S, 0,90 para E e no mínimo 0,70 para VPP e VPN.

Visando explorar diferenças na validade preditiva para PC, quando se usa o critério $\geq 13$ pontos de risco, como proposto pelas autoras do teste ${ }^{18}$, ou $\geq 10$ pontos, como proposto em outros estudos ${ }^{5}$, foram realizados cálculos independentes para cada um desses valores. Além de examinar a validade preditiva para PC, foram incluídos, em um segundo momento, os dados de criançasque não apresentavam diagnóstico de PC, masque apresentavam sinais de atraso no desenvolvimento motor e transtornos da coordenação, aos 6 e 7 anos, quando avaliadas por meio de testes de coordenação motora. Para maiores detalhessobre ostestesutilizadospara o diagnóstico de transtornos da coordenação motora, ver estudos anteriores ${ }^{28,29}$.

\section{RESULTADOS}

A caracterização da amostragem está apresentada na Tabela 1. Ascriançasforam distribuídasem dois grupos, de acordo com o diagnóstico: desenvolvimento típico ou portadora de PC. Foram encontrados 13 (14,6\%) casos de PC (5 tetraparesia espástica, 5 diplegia, 1 hemiparesia direita, 1 hemiparesia esquerda e 1 caso de retardo mental com sinais piramidais).

O exame da estabilidade dos escores do MAI aos 4 e 8 meses de idade corrigida, feito pelo cálculo da correlação (Pearson) entre os escores obtidosnas duasidades, com resultado $r=0,71$ ( $p>$ $0,00)$, indica associação moderada entre osescores obtidosnas duas idades. Na Tabela 2, são apresentados os valores usadospara o cálculo da validade preditiva para PC, usando como ponto de corte escores de risco $\geq 13$ pontos.

Tabela 1. Características da amostragem.

\begin{tabular}{lcc}
\hline & Desenvolvimento Normal & Portadoras de PC \\
\hline Número total de crianças & 76 & 13 \\
Sexo feminino & 44 & 02 \\
Sexo masculino & 32 & 11 \\
Idade gestacional & 27 a 32 semanas & 27 a 32 semanas \\
Idade gestacional média & $29,78( \pm 0,49)$ & $29,46( \pm 1,56)$ \\
Peso ao nascimento & 660 a $1480 \mathrm{~g}$ & 650 a $1430 \mathrm{~g}$ \\
Peso médio ao nascimento & $1.147,10( \pm 220,65)$ & $1.106,54( \pm 241,53)$ \\
Escores do MAl aos 4 meses & 0 a 18 pontos de risco & 2 a 31 pontos de risco \\
Escores médio do MAl aos 4 meses & $4,39( \pm 4,5)$ & $16,77( \pm 7,671)$ \\
Escores do MAl aos 8 meses & 0 a 34 pontos de risco & 8 a 57 pontos de risco \\
Escores médio do MAl aos 8 meses & $6,13( \pm 6,09)$ & $32,31( \pm 14,87)$ \\
\hline
\end{tabular}


A Tabela 3 apresenta um resumo das estimativas de validade preditiva exclusivamente para PC, considerando escores de risco $\geq 13$ pontose $\geq 10$ pontos. A mesma Tabela mostra também os resultados da validade preditiva quando se inclui no cálculo tanto as crianças portadoras de PC, quanto aquelas que apresentaram sinais de transtorno da coordenação motora aos 6 e 7 anos de idade. Observa-se melhor sensibilidade na detecção de PC quando se usa o critério de 10 pontos, mas melhor especificidade para o critério de 13 pontos.

Ao incluir na análise as crianças portadoras de PC e as que apresentavam transtorno da coordenação, quando se utilizou valores $\geq 13$ pontos de risco como ponto de corte, novamente a sensibilidade foi baixa aos 4 , aos 8 e na combinação de 4 e 8 meses, aumentando quando se considerou 10 pontos de risco como ponto de corte. A especificidade foi de moderada a alta em qualquer dasidades, independente do ponto de corte utilizado, variando entre 0,84 e 0,97 . Osresultados do VPP foram superio- res aos encontrados quando se considera apenas crianças com PC, variando de 0,58 a 0,82

\section{DISCUSSÃO}

Os resultados deste estudo indicam que o MAI tem utilidade clínica na detecção precoce de PC, em crianças pré-termo brasileiras. No entanto, o teste mostrou ser mais específico para discriminar crianças com desenvolvimento normal do que para detectar crianças com diagnóstico de PC. A freqüência de $14,6 \%$ de casos de PC encontrada neste estudo (Tabela 1) é similar à observada em outrostrabalhos com o MAI ${ }^{5,24}$, mas é mais alta do que osvalores de $4,4 \%$ e $8,6 \%$ reportados em estudos brasileiro $s^{9,28}$. Esse fato provavelmente está relacionado à amostragem, que inclui grande número de recémnascidos pré-termo extremos, os quais têm maior probabilidade de apresentar seqüelas neuromotoras. Osoutros estudos brasileirosincluíram recémnascidos com até 36 semanas.

Tabela 2. Distribuição de crianças com escore de risco no $\mathrm{MAl} \geq 13$ pontos e diagnóstico de paralisia cerebral

\begin{tabular}{lcccccc}
\hline \multirow{2}{*}{ Diagnóstico } & \multicolumn{4}{c}{ Escores do MAl } \\
\cline { 2 - 7 } & \multicolumn{3}{c}{$\geq 13$ pontos de risco } & \multicolumn{3}{c}{$<13$ pontos de risco } \\
\cline { 2 - 8 } & $4 \mathrm{~m}$ & $8 \mathrm{~m}$ & 4 e 8m & $4 \mathrm{~m}$ & $8 \mathrm{~m}$ & 4 e 8m \\
\hline Portador de PC & 8 & 12 & 8 & 5 & 1 & 1 \\
Não portador de PC & 7 & 8 & 3 & 69 & 68 & 62 \\
Total & 15 & 20 & $11^{*}$ & 74 & 69 & $63^{*}$ \\
\hline PC, paralisia cerebral. *Aos 4 e 8 meses, algumas crianças não tiveram escore $\geq 13$ pontos de risco, nas duas
\end{tabular}
avaliações, por isso não foram incluídas.

Tabela 3. Número de pontos de risco e validade preditiva do MAI para crianças com PC e ADNPM

\begin{tabular}{|c|c|c|c|c|}
\hline & $\begin{array}{c}\mathrm{MAI} \geq 13 \\
\text { predição de } \mathrm{PC}\end{array}$ & $\begin{array}{c}\mathrm{MAI} \geq 10 \\
\text { predição de PC }\end{array}$ & $\begin{array}{c}\text { MAI } \geq 13 \\
\text { predição de } \\
\text { PC/ADNPM }\end{array}$ & $\begin{array}{l}\text { MAI } \geq 10 \\
\text { predição de } \\
\text { PC/ADNPM }\end{array}$ \\
\hline \multicolumn{5}{|c|}{4 meses } \\
\hline VPP & 0,53 & 0,48 & 0,60 & 0,58 \\
\hline VPN & 0,93 & 0,98 & 0,86 & 0,92 \\
\hline $\mathrm{S}$ & 0,61 & 0,92 & 0,47 & 0,74 \\
\hline E & 0,91 & 0,83 & 0,91 & 0,86 \\
\hline \multicolumn{5}{|c|}{8 meses } \\
\hline VPP & 0,60 & 0,43 & 0,70 & 0,59 \\
\hline VPN & 0,98 & 0,79 & 0,93 & 0,95 \\
\hline $\mathrm{S}$ & 0,92 & 0,92 & 0,74 & 0,84 \\
\hline E & 0,89 & 0,79 & 0,91 & 0,84 \\
\hline \multicolumn{5}{|c|}{4 e 8 meses } \\
\hline VPP & 0,73 & 0,65 & 0,82 & 0,76 \\
\hline VPN & 0,98 & 1,00 & 0,92 & 0,96 \\
\hline $\mathrm{S}$ & 0,89 & 1,00 & 0,64 & 0,87 \\
\hline$E$ & 0,95 & 0,90 & 0,97 & 0,93 \\
\hline
\end{tabular}

PC, paralisia cerebral; ADNPM, atraso no desenvolvimento neuropsicomotor; VPP, valor de predição positivo; VPN, valor de predição negativo; S, Sensibilidade; E, Especificidade. 
As crianças portadoras de PC obtiveram maior número de pontos de risco no MAI. Além disso, o número de pontos de risco dessas crianças sobe acentuadamente dos 4 para os 8 mesese há apenas discreta elevação na pontuação para as crianças com desenvolvimento normal. Uma pequena elevação nos escores entre as duas idades é esperada, pois o MAl aos 8 meses possui mais itens a serem avaliados, uma vez que nessa idade a criança apresenta mais atividades voluntárias (i.e., alcance, preensão, rolar, sentar e progressão em prono) e reações automáticas (i.e., reações de equilíbrio e proteção). Todavia, o aumento acentuado na pontuação das crianças com PC sugere que aos 8 meses de idade corrigida (i.e., no mínimo 10 meses de idade cronológica para a amostragem em questão), muitas crianças apresentam sinais neuromotores típicos da PC, detectadospelo MAI, o que aumenta o número de pontos de risco. Esse aumento no número de pontos de risco aos 8 meses, nas crianças com PC possivelmente influenciou o valor da correlação entre os escores obtidos aos 4 e aos 8 meses, que ficou na faixa moderada $(r=0,71)$, indicando certa instabilidade dos escoresnas duasidades. Esta correlação, apesar de estar abaixo dos 0,80 esperado para indicar estabilidade dos escores, é consistente com os valores de 0,76 a 0,79 descritos para confiabilidade teste-reteste do $\mathrm{MAI}^{18,20}$, especialmente quando se considera que, no presente estudo, houve um intervalo de quatro mesesentre as duas testagens.

Examinando o efeito dos diferentes pontos de corte nosíndices de validade preditiva, observa-se que, ao contrário de estudos mais recentes ${ }^{5,24}$, o ponto de corte $\geq 13$ pontos de risco (Tabela 3), como sugerido no manual do $\mathrm{MAI}^{18}$, foi o que apresentou melhor combinação de sensibilidade e especificidade para a amostragem brasileira, nas duas idades estudadas. Nota-se que, apesar da sensibilidade do MAI aos 4 meses ser melhor quando se usa o critério de $\geq 10$ pontos de risco (Tabela 3), a especificidade e o valor de predição positiva são menores, com aumento de falso positivos, o que pode ter conseqüênciasnegativas para $17 \%$ de crianças, incorretamente consideradassuspeitas de terem PC. Em consonância com a literatura ${ }^{24}$, a validade preditiva para crianças aos 8 meses de idade corrigida foi melhor do que aos 4 meses, independentemente do ponto de corte utilizado (Tabela 3). Como esperado, a combinação do MAl aos 4 e 8 meses foi a que apresentou melhor validade preditiva.

Os índices calculados, exceto VPP, estão quase todos dentro do valores propostos por Glascoe e colaboradore ${ }^{27}$ para se afirmar a validade do MAI na detecção precoce de PC, para as crianças do presente estudo. Observa-se que os VPP só estão acima do limite quando se considera a avaliação aos 4 e 8 meses $(0,73)$. Os baixos valores de VPP indicam que o MAl apresenta alto índice de falso-positivos, ou seja, um escore de risco $\geq 13$ pontos pode não significar que a criança seja portadora de PC. Na verdade, de acordo com a idade de aplicação do teste e o ponto de corte utilizado, há probabilidade de 27 a 52\% da criança não ter diagnóstico final de PC (Tabela 3). Infelizmente, isso pode fazer com que uma criança que apresentará desenvolvimento motor adequado seja encaminhada, desnecessariamente, a programas de intervenção, com ônuspara a criança, sua família e para o Estado. Por outro lado, a alta especificidade, indica que o MAl é útil para prever desenvolvimento normal, ou seja, para a criança com pontuação $\leq 10$ pontos de risco, podemos tranqüilizar os pais, pois há 79 a $100 \%$ de probabilidade do desenvolvimento ser normal.

Para as crianças com diagnóstico de PCe/ou problemas de coordenação motora, o MAl apresentou melhor sensibilidade com o critério $\geq 10$ pontos de risco, independentemente da idade em que foi realizada a avaliação. Porém, a especificidade é meIhor quando se adota o ponto de corte $\geq 13$ pontos. O alto VPP indica que $82 \%$ das crianças que tiveram pontuação acima de 13 pontos, aos 4 e 8 meses, estão sujeitas a apresentar algum tipo de transtorno motor, o que pode incluir problemas de coordenação motora na idade escolar. Tais dados ressaltam a importância de se acompanhar o desenvolvimento dessas crianças até a entrada na escola.

\section{CONCLUSÃO}

O MAI apresentou valores moderados de validade preditiva e tem melhor utilidade na predição de PC para crianças brasileiras nascidas prematuras, quando aplicado aos 8 meses de idade, com ponto de corte $\geq 13$ pontos de risco. A alta especificidade e os baixos VPP alertam para se ter cautela na interpretação dosescores de risco do MAI. Ou seja, crianças com escoresabaixo de 10 pontos provavelmente apresentarão desenvolvimento normal, mas os escores $\geq 13$ pontos de risco não são indicativos seguros de evolução para PC. Como as crianças que apresentaram escores acima do ponto de corte nas duas avaliações, aos 4 e 8 meses, têm mais chances de apresentar PC, recomenda-se pelo menos duas avaliações consecutivas, antes de se considerar a possibilidade de seqüela neuromotora. 
Apesar do objetivo do MAl ser a detecção de PC, este estudo mostra que os escores de risco podem ser úteis para detectar problemasmotores mais leves, que aparecem na idade escolar, dado ainda não documentado em outrostrabalhos. Nesse caso, o uso de diferentes pontos de corte depende dos objetivos do programa: se a meta é detecção de $\mathrm{PC}$, um ponto de corte mais restritivo ( $\geq 13$ pontos) é mais útil clinicamente. Se a meta é também detectar quadrosmotores maisleves, pode-se usar um ponto de corte mais inclusivo ( $\geq 10$ pontos).

Embora com algumas limitações, o MAI oferece ao terapeuta uma estrutura objetiva para a avaliação do bebê. É surpreendente que escores obtidos aos 4 e 8 meses possam ter algum valor para prever, mesmo que de maneira grosseira, transtornos da coordenação motora observados na criança em idade escolar. Pode-se concluir que crianças com escores de risco acima de 10 pontos, aos 4 e 8 meses de idade, são suspeitas para o aparecimento de transtornos motores e devem estar inseridas em programaslongitudinais de acompanhamento do desenvolvimento, até a idade escolar. Uma vez que o presente estudo analisou apenas os padrões de escore de crianças prematuras nascidas com até 32 semanas de idade gestacional, trabalhos futuros devem verificar a validade preditiva do MAI para crianças brasileiras, que nasceram com mais de 32 semanas de idade gestacional ou recém-nascidos a termo expostos a outros fatores de risco perinatais. Ressaltamosainda, que o exame neurológico, quando realizado por profissional experiente, também permite a detecção precoce de PC, embora algumas alterações leves possam ser transitórias. Seria interessante aplicar o MAI, concomitante à avaliação neurológica, para comparar a eficácia dos dois métodos na detecção precoce de transtornos neuromotores.

Agradecimentos - Agradecemosà equipe do ACIAR, pelo suporte ao trabalho interdisciplinar e, principalmente, às crianças e familiares que tornaram possível a condução deste estudo.

\section{REFERÊNCIAS}

1. Halloway E. Como favorecer o entretenimento progenitor-lactente na Unidade de Terapia Intensiva Neonatal. In Parham LD, Fazio LS (eds). A recreação na terapia ocupacional pediátrica. São Paulo: Editorial Santos, 2000:171-183.

2. Levine MS. Cerebral palsy diagnosis in children over age 1 year: standard criteria. Arch Phys Med Rehabil 1980;61:385-389.

3. Harris SR. Early neuromotor predictors of cerebral palsy in lowbirthweight infants. Dev Med Child Neurol 1987;29:508-519.
4. Darrah J, Piper MC, Byrne PJ, Warren S. The utilization of the Movement Assessment of Infants risk profile with preterm infants. Phys Occ Ther Pediatrics 1991;11:1-11.

5. Swanson MW, Bennett FC, Shy KK, Whitfield MF. Identification of neurodevelopmental abnormality at four and eight months by the Movement Assessment of Infants. Dev Med Child Neurol 1992;34:321337.

6. Rutter N. The extremely preterm infant. Br J Obstet Gynecol 1995;102:682687.

7. Goulart AL, Barros MCM, Azevedo MF, Domingues SS, Meyerhof P, Vilanova LCP. Crescimento e desenvolvimento do recém-nascido prétermo. Acta Paul Enf 1996;9:82-88.

8. Allen MC, Alexander GR. Using motor milestones as a multistep process to screen preterm infants for cerebral palsy. Dev Med Child Neurol 1997;39:12-16.

9. Mello RR, Dutra MVP, Silva KS, Lopes JM. Valores de predição da avaliação neurológica e ultra-sonográfica cerebral neonatal em relação ao desenvolvimento de prematuros de muito baixo peso. Rev Saúde Pública 1998;32:420-429.

10. DeGroot L. Posture and motility in preterm infants. Dev Med Child Neurol 2000;42:65-68.

11. Cans C. Surveillance of cerebral palsy in Europe: a collaboration of cerebral palsy surveys and registers. Dev Med Child Neurol 2000;42:816-824.

12. Magalhães LC, Araújo AR, Paixão ML, Mancini MC, Barbosa VM. Influência de fatores de risco biológico nos escores de um teste para detecção de paralisia cerebral em crianças pré-termo. Temas 2001;10:5-12.

13. Dargassies SAS. Méthode d'examen neurologique du nouveau-né. Études Neonat 1954;3:101-124.

14. Amiel-Tison C. Neurological evaluation of the maturity of newborn infants. Arch Dis Child 1968;43:89-93.

15. Prechtl HFR, Einspieler C, Cioni G, Bos AF, Ferrari F, Sontheimer D. An early marker for neurological deficits after perinatal brain lesions. Lancet 1997;349:1361-1363.

16. Dubowitz L, Dubowitz V. The neurological assessment of the full-term newborn infant. Clinics in Developmental Medicine. London: Spastics International Medicinal Publications,1981.

17. Campbell SK, Kolobe THA, Osten ET, Girolani GL, Lemke M. Test of Infant Movement Performance. Research edition. Chicago: University of Illinois, 1995.

18. Chandler LS, Andrews MS, Swanson MW. Movement Assessment of Infants: a manual. Rolling Bay, A: Authors, 1980.

19. Schneider J, Lee W, Chasnoff IJ. Field testing of the Movement Assessment of Infants. Phys Ther 1988;68:321-327.

20. Harris SR, Haley SM, Tada WL, Swanson MW. Reliability of observational measures of the Movement Assessment of Infants. Phys Ther 1984;64:471-475.

21. Haley SM, Harris SR, Tada WL, Swanson MW. Item reliability of the Movement Assessment of Infants. Phys Occ Ther Pediatr 1986;6:21-39.

22. Campbell SK. Using standardized tests in clinical practice. In Campbell S; Russell EC (eds). Topics in Pediatrics - Lesson 11. Alexandria: American Physical Therapy Association, 1990:1-10.

23. Washington K, Deitz JC. Performance of full-term 6-month-old infants on the Movement Assessment of Infants. Ped Phys Ther 1995;7:65-74.

24. Darrah J, Piper M, Watt MJ. Assessment of gross motor skills of at-risk infants: predictive validity of the Alberta Infant Motor Scale. Develop Med Child Neurol 1998;40:485-491.

25. Fetters L, Tronick EZ. Discriminate power of the Alberta Infant Motor Scale and the Movement Assessment of Infants for prediction of Peabody Gross Motor Scale Scores of infants exposed in utero to cocaine. Ped Phys Ther 2000;12:16-23.

26. Piper MC, Darrah J. Motor assessment of the developing infant. Philadelphia: W.B. Saunders, 1994.

27. Glascoe FP, Byrne KE, Ashford LG, Johnson KL, Chang B, Strickland B. Accuracy of the Denver-II in developmental screening. Pediatrics 1992;89:1221-1224.

28. Magalhães LC, Barbosa VM, Paixão ML, Figueiredo EM, Gontijo APB. Acompanhamento ambulatorial de recém nascidos de alto risco: características da população atendida e incidência de seqüelas funcionais. Rev Paul Pediatr 1998;6:191-196.

29. Magalhães LC, Wendling PC, Paixão ML, Mancini MC, Barbosa VM Estudo comparativo sobre o desempenho perceptual e motor na idade escolar em crianças nascidas pré-temo e a termo. Arq Neuropsiquiatr 2003;61:250-255. 